\title{
GUIDANCE AND CONTROL FOR MARS ATMOSPHERIC ENTRY: ADAPTIVITY AND ROBUSTNESS 1
}

\author{
Wei-Min Lu * and David S. Bayard ** \\ - AMRL, AE5/282, IBM Corporation, San Jose, CA 95193; \\ Email: wmivOus.ibm.com \\ ** JPL 198-326, CalTech, Padadena, CA 91109
}

\begin{abstract}
In this paper, we address the atmospheric entry guidance and control problem for Mars precision landing. The guidance and control design is based on the principle of tracking a reference drag versus velocity profile in the entry flight corridor, which is determined by physical constraints of the flight. An integrated adaptive/robust control approach to atmospheric entry guidance and control is introduced to deal with different uncertainties.
\end{abstract}

Keywords: Aerospace Systems, Adaptive Control, Guidance and Control, Nonlinear Systems, Planetary Entry, Robust Control

\section{INTRODUCTION}

To facilitate guidance, navigation, and control design for Mars precision landing, the Mars landing process is divided into three phases, i.e., atmospheric entry phase, parachute phase, and terminal landing phase. In this study, we only focus on guidance and control design for the entry phase, i.e., the phase from atmospheric entry of Mars orbiter to parachute deployment. Feedback aerodynamic maneuvering during the entry phase is used to reduce the error to a required level at the parachute deployment. Hindering the achievement of this goal are uncertainties in the guidance and control systems. The main sources of the uncertainties include

\footnotetext{
1 Partially supported by IBM and NASA; part of the work was done when the first author was with JPL
}

winds/gusts, air density dispersions, and measurement and knowledge errors; in addition, some simplification assumption on mathematical model made to facilitate the control design also generates a gap the real system and the employed model, which is conveniently treated as uncertainty. Basically, the above uncertainties are classified as external disturbances and parametric uncertainty.

In this paper, we will address the guidance and control problem from the view point of adaptive and robust control design. The following questions will be answered to provide an integrated design method: given an a priori error bound, does there exist any guidance and control law to achieve an error level not greater than the given bound under all admissible disturbances and vehicle evolution parameter changes, and how does one design such 
a control law? In particular, we consider the longitudinal landing guidance following McEneaneyMease's approach [6]. The objective is to achieve a certain specified range during entry by control of the lift/drag ratio component in position-velocity plane, which is achieved by modulation of the Mars landing vehicle's angle of attack and/or bank angle. The guidance approach is based on the principle of tracking a reference drag-velocity profile in the entry flight corridor, which is determined in the drag-velocity plane by physical constraints of the flight; those constraints include the limitations of the thermal protection system surface temperatures, normal load factor, dynamic pressure, and equilibrium glide flight (see [2] for details). A guidance approach based on drag reference tracking has been used in the NASA Space Shuttle re-entry guidance law $[2,3]$. Basing guidance and control law on drag is advantageous because drag is essentially directly measured by the accelerometer, and the range to be flown during entry can be decided by the drag acceleration profile maintained throughout the entry flight and can be predicted using simple analytical techniques.

There exists several guidance and control techniques under ideal conditions, i.e., assuming perfect measurement and no uncertainty $[2,6,7]$, but without guarantee for error tolerance level due to disturbances. In this paper, basing on previous guidance and control results, we will develop an integrated guidance and control law to minimize the impact of uncertainties. This 2-part paper is organized as follows: in section 2, an uncertain drag dynamical model is derived from the basic equations of motion with the consideration of atmospheric disturbances and some operating parameter variations; the guidance and control problem for Mars atmospheric entry is formulated as a problem of tracking a drasvelocity profile. In section 3 , the guidance and control problem is solved as an adaptive disturbance rejection problem where the parametric uncertainty and atmospheric disturbances are treated separately so as to improve system performance.

\section{UNCERTAIN DYNAMICS OF MARS ATMOSPHERIC ENTRY}

The objective of the longitudinal guidance is to control the range to be flown during entry, which is a function of the drag-velocity profile [2]. The main idea for the guidance strategy is to control the vehicle to follow a given drag-velocity profile.

\subsection{Equations of Motion}

The equations of translational motion used in this paper are developed in a coordinate system with one axis oriented along the Mars-relative velocity vector, one axis perpendicular to the plane formed by the position and Mars relative velocity vectors, and the third axis completing the right-hand coordinate system. The Mars-relative translational state of the landing vehicle is represented by the variables $R$ (range), $h$ (altitude), $V$ (velocity relative to the Mars), $\gamma$ (flight path angle), and $\psi$ (heading angle). These equations of motion are as follows [10]:

$$
\begin{gathered}
\dot{R}=V \cos \gamma \\
\dot{h}=V \sin \gamma \\
\dot{V}=-D-g \sin \gamma \\
\dot{\gamma}=\frac{1}{V}\left(\left(\frac{V^{2}}{r}-g\right) \cos \gamma+L \cos \sigma\right) \\
\dot{\psi}=\frac{1}{V \cos \gamma}\left(-\frac{V^{2}}{r} \cos ^{2} \gamma \tan \phi \sin \psi+L \sin \sigma\right)(5)
\end{gathered}
$$

where $\sigma$ is the bank (or roll) angle and $\phi$ is vehicle Mars-relative latitude, $r$ the distance between the lander and the center of Mars, $g=\mu / r^{2}$ with $\mu$ the Martian GM is the Martian gravitational acceleration. These equations of motion neglect the Coriolis and centripetal accelerations due to the Mars' rotation because they are relatively small. If the relative motion of the atmosphere to Mars is ignored, the specific drag and lift are given by the following:

$$
D=\frac{1}{2} \rho \frac{S}{m} C_{D} V^{2} ; L=\frac{1}{2} \rho \frac{S}{m} C_{L} V^{2},
$$

where $C_{D}$ and $C_{L}$ are drag and lift coefficients, respectively; they change with the change of the angle of attack and the magnitude of the velocity. An exponential atmospheric density $\rho$ model is used in the following.

\subsection{Drag Reference and Range Prediction}

For the Mars atmospheric entry, we employ a drag profile in the drag acceleration / Mars-relative velocity plane. The drag profile is chosen to stay 
within the entry flight corridor due to some physical constraints and to minimize the accumulate aerodynamic heat load (see [2] for details). A typical drag profile, $D_{r}=f(V)$, is shown in Fig. 1, composed

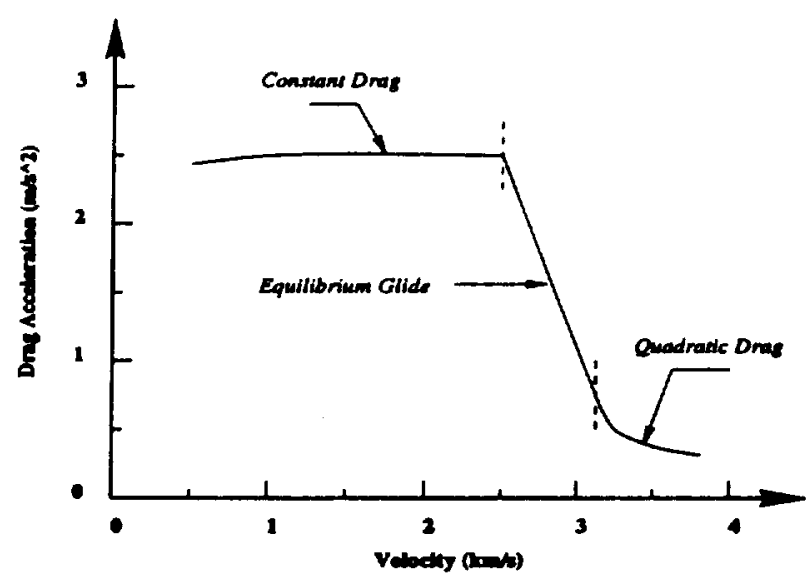

Fig. 1. Typical Drag-Velocity Profile

of a quadratic drag segment, an equilibrium glide (linear) segment, and a constant drag segment $[2,6]$. Each of the segments is taken as a general quadratic profile: $f(V)=c_{0}+c_{1} V+c_{2} V^{2}$. The objective for the entry guidance law is to achieve a desired range at a certain specified velocity. In fact, the range can be approximated by the following relation:

$$
R(V)=-\int_{V_{0}}^{V} \frac{V}{f(V)+g \sin \gamma} d V \approx-\int_{V_{0}}^{V_{f}} \frac{V}{f(V)} d V
$$

at the earlier stage of the flight as the flight path angle is small. During the latter part of the entry when the landing vehicle is at lower speed, one can use an energy variable $E=g h+\frac{1}{2} V^{2}$ to replace the velocity variable $V$ in the drag expression [2]. Therefore, the range to be flown is determined by the drag-velocity (or drag-energy) profile. In the following, the control and guidance law is designed so that the actual drag will track the given dragvelocity profile.

\subsection{Drag Dynamics with Uncertainty}

To facilitate the control design for entry guidance based on the drag-velocity profile, one should directly consider the drag dynamics, instead of the dynamical equations considered earlier in (1)(5). We are particularly interested in the impact of the atmospheric uncertainties, including surface winds/gusts and atmospheric density dispersions.

\section{Impact of Atmospheric Uncertainties}

The atmospheric uncertainties considered in the following include the air density dispersion and the winds/gusts. When uncertainties are presented, $D$ and $L$ in the equations of motion are not drag and lift, rather they are the aerodynamic forces parallel and perpendicular in the plane of symmetry to the vehicle velocity vector relative to Mars, respectively. The air flow velocity due to winds and gusts can be represented as $V_{w}=V+\Delta V$, where $V$ is the velocity of the landing vehicle relative to Mars, $\Delta V$ is the disturbance due to the winds. The drag and lift coefficients change with the change of the angle of attack as well as the magnitude of velocity. The influence of the surface winds/gusts, which are viewed as disturbances, can also contribute to the changes in specific drag and lift coefficients, in which case:

$$
C_{D}=C_{D}^{0}+\Delta C_{D}, \quad C_{L}=C_{L}^{0}+\Delta C_{L}
$$

where $C_{D}^{0}$ and $C_{L}^{0}$ are the actual drag and lift coefficients, and $\Delta C_{D}$ and $\Delta C_{L}$ are the corresponding deviations due to the above treatment.

The atmospheric density can be modeled as the ideal exponential density model (8) plus uncertain dispersion $\Delta \rho$ (see Fig. 2), i.e., it can be well represented by the following:

$$
\rho=\rho_{0} e^{-\frac{h}{h}}+\Delta \rho \text {. }
$$

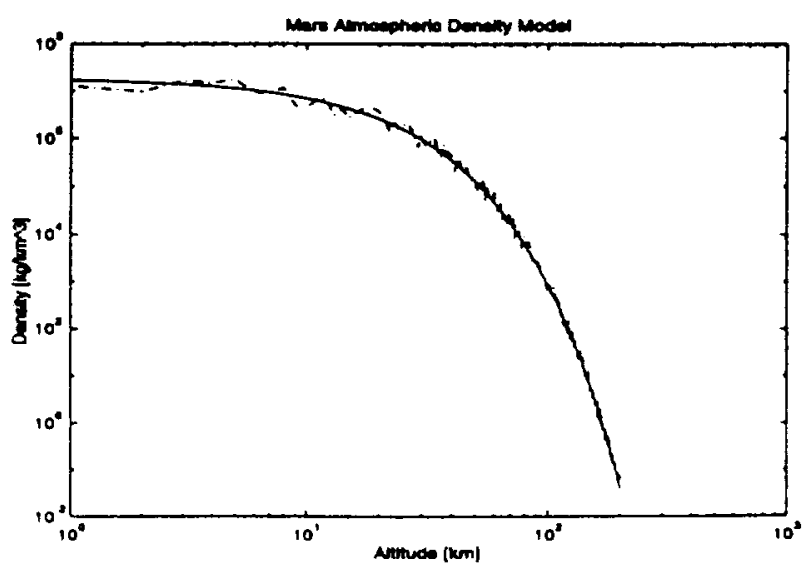

Fig. 2. Mars Atmospheric Density Dispersion 
The influence of the atmospheric uncertainties contributes to the changes in specific drag and lift, in which case: $D=D_{0}+\Delta D$ and $L=L_{0}+\Delta L$, where $D_{0}$ and $L_{0}$ are drag and lift without disturbances, and $\Delta D$ and $\Delta L$ are the resulting perturbations.

From the density model (7), one has that

$$
\frac{\dot{\rho}-\Delta \dot{\rho}}{\rho-\Delta \rho}=-\frac{\dot{h}}{H},
$$

or $\frac{\dot{e}}{p}=-\frac{\dot{h}}{H}+\delta_{d}$ for some $\delta_{d}$.

From the drag formulation, one has

$$
\frac{\dot{D}}{D}=\frac{\dot{\rho}}{\rho}+2 \frac{\dot{V}_{f}}{V_{f}}+\frac{\dot{C}_{D}}{C_{D}}=-\frac{\dot{h}}{H}+2 \frac{\dot{V}}{V}+C+\delta_{a} \cdot(8)
$$

where $C:=\frac{C_{a}^{0}}{C_{D}^{0}}$ and $\delta_{a}=$

$$
\delta_{d}+2 \frac{\Delta \dot{V} V-\Delta V \dot{V}}{(V+\Delta V) V}+\frac{\Delta \dot{C}_{D} C_{D}^{0}-\Delta C_{D} \dot{C}_{D}^{0}}{\left(C_{D}^{0}+\Delta C_{D}\right) C_{D}^{0}}
$$

\section{Uncertain Drag Dynamical Model}

Now we are ready to derive the drag dynamics for control design. The control of the translational motion is performed by adjusting $u:=\frac{L}{D} \cos \sigma$, which can be achieved by the modulation of either angle of attack or the bank angle.

In the following, we assume the gravity acceleration $g=\mu / \bar{r}$ with $\bar{r}$ being the reference radius is constant. The actual variation of $g$ can be taken as a part of the disturbance to be discussed later.

From the equation (2), (3), and (4), one has

$$
\begin{gathered}
\ddot{h}=\frac{d}{d t}(V \sin \gamma)=V \cos \gamma \dot{\gamma}+\dot{V} \sin \gamma \\
=\left(\frac{V^{2}}{\bar{r}}-g\right)-D \frac{\dot{h}}{V}-\frac{V^{2}}{\bar{r}}\left(1-\frac{r}{\bar{r}} \cos ^{2} \gamma\right)+D \cos \gamma
\end{gathered}
$$

With the consideration of atmospheric uncertainties, from (8), one has

$$
\left(1+\frac{2 g H}{V^{2}}\right) \dot{h}=-H\left(\frac{\dot{D}}{D}+2 \frac{D}{V}-C-\delta_{a}\right) .
$$

Take derivatives on both sides, one has

$$
\left(1+2 \frac{g H}{V^{2}}\right) \ddot{h}+6 \frac{g H D}{V^{3}} \dot{h}+4 \frac{g^{2} H \sin ^{2} \gamma}{V^{2}}
$$

$$
=-H\left(\frac{\ddot{D}}{D}-\frac{\dot{D}^{2}}{D^{2}}+2 \frac{\dot{D}}{V}+2 \frac{D^{2}}{V^{2}}-\dot{C}-\dot{\delta}_{a}\right) .
$$

From (10), one can solve $\dot{h}$; replace the $\dot{h}$ and $\ddot{h}$ defined by (9) in (11), one has the following equation:

$$
\begin{aligned}
\ddot{D}+ & g_{0}(D, \dot{D}, V)+g_{1}(D, \dot{D}, V, u) \theta \\
& =w+g_{2}(D, \dot{D}, V) u,
\end{aligned}
$$

for some smooth functions $g_{0}, g_{1}$, and $g_{2}$ of $\dot{D}, D$, and $V$, where $\theta$ is a parameter vector defined by

$$
\theta=\left[\begin{array}{c}
C \\
\dot{C} \\
1-\frac{r}{\bar{r}} \cos ^{2} \gamma \\
1-\cos \gamma \\
\sin ^{2} \gamma
\end{array}\right]
$$

$w$ is the combined disturbances:

$$
w=-\frac{4 g H D^{2}}{V\left(V^{2}+2 g H\right)} \delta_{a}+D \dot{\delta}_{a} ;
$$

On the other hand, from (3) and (2), one has

$$
\begin{gathered}
\dot{V}=-D-g \sin \gamma=-D-g \frac{\dot{h}}{V} \\
=\xi_{0}(D, \dot{D}, V)+\xi_{1}(D, \dot{D}, V) C+\xi_{2}(D, \dot{D}, V) \delta_{a}(14) \\
=: \xi\left(D, \dot{D}, V, C, \delta_{a}\right),
\end{gathered}
$$

for smooth functions $\xi_{0}, \xi_{1}$, and $\xi$ of $D, \dot{D}, V, C$, and $\delta_{a}$.

The above equations give a model for drag dynamics with uncertainty. Note that the model (12)-(14) is fairly general to cover many other sources of uncertainties. In particular, the measurement noises, estimation errors, as well as the gravitational acceleration variations can be conveniently considered (9)s part of the combined disturbance $w$. If we take $x_{1}=D, x_{2}=\dot{D}$, and $x_{3}=V$, then complete dynamics governing the drag evolution is as follows:

$$
\left\{\begin{aligned}
\dot{x}_{1}= & x_{2} \\
\dot{x}_{2}= & -g_{0}\left(x_{1}, x_{2}, x_{3}\right)-g_{1}\left(x_{1}, x_{2}, x_{3}, u\right) \theta \\
& +w+g_{2}\left(x_{1}, x_{2}, x_{3}\right) u \\
\dot{x}_{3}= & \xi\left(x_{1}, x_{2}, x_{3}, C, \delta_{a}\right) \\
z= & x_{1}
\end{aligned}\right.
$$

In the following, we will use the above dynamical equation to design a guidance law. 


\section{GUIDANCE AND CONTROL DESIGN}

\subsection{Guidance as a Tracking Problem with Disturbance Rejection}

As the drag profile is given by the range-velocity requirement, to reach certain range requirement, it is sufficient for the drag governed by the dynamics (12)-(14) to follow the corresponding drag-velocity profile in the presence of disturbances. Therefore, the guidance problem can be formulated as a tracking problem with disturbance rejection.

Guidance Problem: Consider the uncertain system (15), suppose $\theta, \in \theta$, where $\theta$ is a convex closed bounded admissible parameter set. Given a dragvelocity profile, $D_{r}=f(V)$; design a feedback controller with measurement of $\dot{D}, D$, and $V$, as well as the profile information, such that for all admissible energy-bounded unknown parameters, $D$ governed by (12) satisfies the following requirements:

- Tracking. if $w=0$,

$$
\lim _{t \rightarrow \infty}\{D(t)-f(V(t))\}=0 .
$$

- Disturbance Rejection:

$$
\int_{0}^{T}\left\|D(t)-D_{r}(t)\right\|^{2} d t \leq \lambda^{2} \int_{0}^{T}\|w(t)\|^{2} d t+\epsilon
$$

for given $\epsilon>0$, and some $\lambda>0$.

In the following section, we will provide an integrated adaptive/robust control approach to deal with the above problem based on the dynamical equation (15). The control architecture is illustrated in Fig. 3. The techniques of linearization, $\mathcal{H}_{\infty^{-}}$ control, and dissipation theory-based adaptive control will be used ([5]).

\subsection{Feedback Linearization}

The starting point for this problem is to design a controller for the disturbance rejection problem with assumption that the parameter $\theta$ is known. Consider system (15), take

$$
u=\frac{g_{0}\left(x_{1}, x_{2}, x_{3}\right)+g_{1}^{0}\left(x_{1}, x_{2}, x_{3}\right) \dot{\theta}+v}{\left(1+\theta_{5}\right) g_{2}\left(x_{1}, x_{2}, x_{3}\right)}
$$

where $v$ is the new input variable. Let $z=\left[\begin{array}{l}z_{1} \\ z_{2}\end{array}\right]$, where

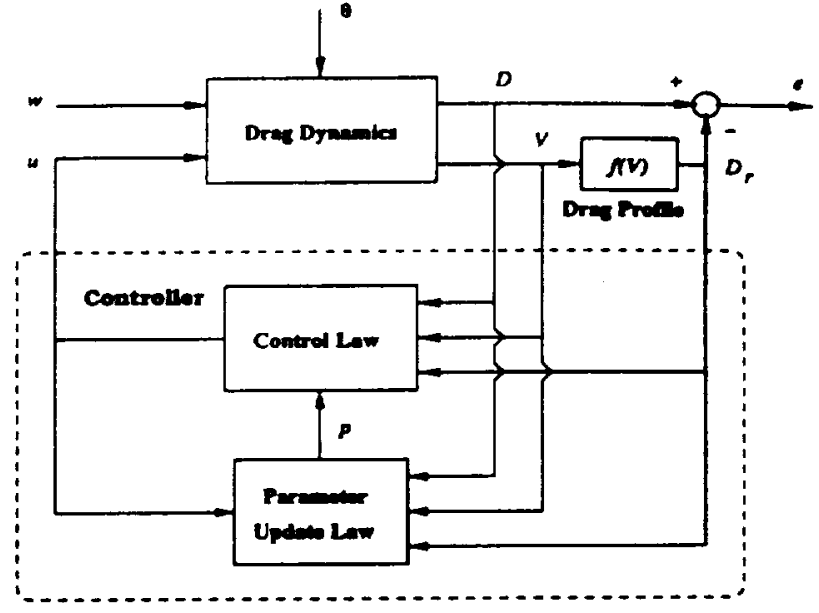

Fig. 3. Adaptive Disturbance Rejection

$$
z_{1}=x_{1}-D_{r}=e, z_{2}=x_{2}-\dot{D}_{r}
$$

then equation (15) can be represented as:

$$
\left\{\begin{array}{l}
\dot{z}=A z+B_{1} d+B_{2} v \\
\dot{x}_{3}=\xi\left(z_{1}+D_{r}, z_{2}+\dot{D}_{r}, x_{3}, C, \delta_{a}\right) \\
e=z_{1}
\end{array}\right.
$$

where $A=\left[\begin{array}{ll}0 & 1 \\ 0 & 0\end{array}\right], B_{1}=B_{2}=\left[\begin{array}{l}0 \\ 1\end{array}\right]$, and $C=$ $\left[\begin{array}{ll}1 & 0\end{array}\right]$. The above (error) system is input-output linear with linear party state $z$; we will use a linear technique to derive the control $v$.

\section{3 $\mathcal{H}_{\infty}$-Control Design}

Consider the linear part of the equation (17) with state $z$. Note that the error system is independent of the state variable $x_{3}=V$ and observable from output $e=z_{1}$. Suppose $P>0$ is a solution to the Riccati equation for the state-feedback $\mathcal{H}_{\infty}$-control problem:

$$
P A+A^{T} P+P\left(\frac{1}{\lambda^{2}} B_{1} B_{1}^{T}-B_{2} B_{2}^{T}\right) P+C^{T} C=0 .
$$

If $\lambda>1$, then $P>0$. In this case, the $\mathcal{H}_{\infty}$-control solution gives feedback controller:

$$
v=F z:=\frac{1}{2} B_{2}^{T} P z .
$$

with $\int_{0}^{T}\left(\|e(t)\|^{2}\right) d t \leq \lambda^{2} \int_{0}^{T}\left(\|d(t)\|^{2}\right) d t$. One can recover the following controller:

$$
u=K\left(\theta ; \ddot{D}_{r}, \dot{D}_{r}, D_{r}, x_{1}, x_{2}, x_{3}\right)
$$




$$
:=\frac{F z-\ddot{D}_{r}+g_{0}\left(x_{1}, x_{2}, x_{3}\right)+g_{1}^{0}\left(x_{1}, x_{2}, x_{3}\right)}{\left(1+\theta_{6}\right) g_{2}\left(x_{1}, x_{2}, x_{3}\right)}
$$

to achieve the disturbance rejection.

\subsection{Adaptive Control Law}

However, as $\theta$ is not known, the above control law can not be directly used. One can use an estimate $p$ of $\theta$ instead, and the control law would be

$$
u=K\left(p ; \ddot{D}_{r}, \dot{D}_{r}, D_{r}, x_{1}, x_{2}, x_{3}\right)
$$

where $p$ is given by an update law:

$$
\dot{p}=\eta(x, u) \text {. }
$$

Therefore, the guidance and control system has the structure as illustrated in Fig. 3. The error equation can be equivalently represented as:

$$
\left\{\begin{array}{c}
\dot{z}=\left(A+B_{2} F\right) z+B_{1} w \\
+\left[\begin{array}{c}
0 \\
g_{1}\left(x_{1}, x_{2}, x_{3}, u\right)
\end{array}\right](p-\theta) \\
\dot{x}_{3}=\xi\left(z_{1}+D_{r}, z_{2}+\dot{D}_{r}, x_{3}, C, \delta_{a}\right) \\
e=z_{1}
\end{array}\right.
$$

where $F$ is a state feedback matrix defined as $F=$ $\frac{1}{2} B_{2}^{T} P$ and $P$ satisfies the following equation:

$$
\begin{gathered}
P\left(A+B_{2} F\right)+\left(A+B_{2} F\right)^{T} P \\
\quad+\frac{1}{\lambda^{2}} P B_{1} B_{1}^{T} P+C^{T} C=0 .
\end{gathered}
$$

If $V(z)=z^{T} P z$, then completion of square implies

$$
\frac{\partial V}{\partial x}\left(\left(A+B_{2} F\right) z+B_{1} w\right) \leq \lambda^{2}\|w\|^{2}-\|e\|^{2}(19)
$$

To design the update law, we take the following function as a storage function [5]:

$$
W(z, p)=V(z)+(p-\theta)^{\mathbf{T}} Q(p-\theta)
$$

where $Q>0$ such that

$$
\max _{p, \theta \in \Theta}\left\{(p-\theta)^{T} Q(p-\theta)\right\} \leq \epsilon .
$$

Therefore, by completion of square, one has

$$
\begin{gathered}
\dot{W}(z, p) \leq \lambda^{2}\|w\|^{2}-\|e\|^{2}+ \\
2(p-\theta)^{T} Q\left(\dot{p}+Q^{-1}\left[0 g_{1}^{T}\left(x_{1}, x_{2}, x_{3}, u\right)\right] P z\right) .
\end{gathered}
$$

Therefore, if one chooses

$$
\dot{p}=\pi_{\Theta}^{Q}\left(p, Q^{-1}\left[0-g_{1}^{T}\left(x_{1}, x_{2}, x_{3}, u\right)\right] P z\right),
$$

where $\pi_{\Theta}^{Q}$ is the vector projection (see [5]), then

$$
(p-\theta)^{T} Q\left(\dot{p}+Q^{-1}\left[0 g_{1}^{T}\left(x_{1}, x_{2}, x_{3}, u\right)\right] P z\right) \leq 0 .
$$

Thus, we have

$$
\int_{0}^{T}\|e\|^{2} d t \leq \lambda^{2} \int_{0}^{T}\left(\|w\|^{2}\right) d t+\epsilon .
$$

Let's look at the tracking issue if $w=0$. We still use $W(z, p)$ as the Lyapunov function. Then with the above adaptive law, one has $\dot{W}(z, p) \leq-\|e\|^{2} \leq 0$. Therefore, $z$ and $p-\theta$ are bounded. It can be shown that $\int_{0}^{\infty}\|e\|^{2} d t<\infty$, and $e(t) \rightarrow 0$ as $t \rightarrow \infty$.

Therefore, the adaptive control law given by (18)(20) achieves adaptive disturbance rejection.

ACKNOWLEDGEMENT: The authors would like to thank D. Farless, and Drs. D. Boussalis, K. Mease, and R. Smith for their helpful inputs.

\section{REFERENCES}

[1] D. Boussalis, "Investigation of Longitudinal Motion of Low-Lift Entry Vehicles," JPL Engi. Memo., No.3456-96-002, JPL, 1996.

[2] J.C. Harpold and C.A. Graves, Jr., J. of Astro. Sciences, Vol.27(3), pp.239-268, 1979.

[3] J.C. Harpold and D. E. Gavert, AIAA J. Guidance, Vol.6(6), pp.442-447, 1983.

[4] R.N. Ingoldby, AIAA J. Guidance and Control, Vol.1(3), pp.189-196, 1978.

[5] W.-M. Lu and A. Packard, "Adaptive $H_{\infty}$ Control of Nonlinear Uncertainty Systems," Proc. 1996 ACC, Albuquerque, NM, 1997.

[6] W.M. McEneaney and K.D. Mease, J. of Astro. Sciences, Vol.39(4), pp.423-445, 1991.

[7] K.D. Mease and J-P. Kremer, AIAA J. Guidance, Contr.. Dynamics, Vol.17(6), pp.13501356, 1994.

[8] R. Smith, "Closed-Loop Aeromaneuvering for a Mars Precision Landing," CCEC Tech. Reporh, No.CCEC-96-0205, UCSB, 1996.

[9] D.G. Tuckness, AIAA J. Spacecraft and Rockets, Vol.32(1), pp.142-148, 1995.

[10] N.X. Vinh, A. Busemann, and R.D. Culp, $\mathrm{Hy}$ personic and Planetary Entry Flight Mechanics, Ann Arbor: University of Michigan Press, 1980. 